\title{
ASSESSING, AGGREGATING AND VISUALISING PRELIMINARY DESIGN INFORMATION MATURITY TO SUPPORT COLLABORATIVE DESIGN
}

\author{
J. T. Brinkmann ${ }^{\otimes}$ and D. C. Wynn \\ The University of Auckland, New Zealand \\ $\bowtie$ jbri364@aucklanduni.ac.nz
}

\begin{abstract}
Engineering projects involve the progressive development of preliminary information until a final design is reached. Appreciating its status may help make better decisions about task sequencing and may reduce unnecessary iteration. We present an approach to 1) elicit this maturity, 2) aggregate several of its facets per subsystem and generate insights for task prioritisation, and 3) overlaying this information in Augmented Reality onto a physical prototype. The progress is discussed and it is proposed that the approach can aid understanding, communication and management of design progress.
\end{abstract}

Keywords: preliminary information, maturity levels, collaborative design, augmented reality (AR), visualisation

\section{Introduction}

Design practitioners often need to share - and base decisions on - immature information. The reasons are either inherent to the interdependence of the design problem, or due to a collaborative concurrent workflow. The effects are intensified if no informal meetings at the colleague's desk are possible, and if concurrency is introduced to coupled activities (Krishnan et al., 1997). If the status of maturity of design information being shared is not explicit and easily visible to all participants ranging from designers to managers, confusion and wasted effort may be caused. For example, sophisticated methods may be applied before the input information is mature enough to justify it.

There could be various benefits if designers were enabled to understand, assess and communicate the various facets of maturity associated with preliminary information in engineering design. With an improved information foundation, easier communication with less misunderstandings is probable. This could lead to less wasted effort or rework, allowing more time to be spent working on relevant aspects of the designs. Hence, increased satisfaction and better designs could be expected - potentially faster and at lower cost. But there are various reasons why the communication of information maturity is not common in engineering practice, ranging from the additional effort of assessing and attaching maturity information during the design process, to challenges of supplying enough context for understanding. Furthermore, the easy use of carry-over or downloaded resources as CAD parts may induce a misleading appearance of finality where in fact these are merely placeholders with a high likelihood of change. Thus motivated, this paper introduces a concept for an approach to assess, aggregate and visualise maturity of preliminary design information with the intent to support engineering design processes. 


\section{Development of a concept for a support approach}

\subsection{Insights from literature}

A literature review was undertaken to explore previous work on maturity of preliminary design information. It was found that there are multiple perspectives and multiple facets of maturity, such as precision, completeness, stability, ambiguity, and many more. Several authors have discussed these perspectives and facets and attempted to operationalise some of them for problem-framing (Schrader et al., 1993) or determining task overlapping strategies (Krishnan et al., 1997; Terwiesch et al., 2002). The related topic of uncertainty in design information has also received significant attention (e.g. Thunnissen, 2005). However, few in-depth taxonomies that organise the multiple facets of design information maturity have been developed, apart from Grebici (2007) and Thunnissen, (2005) who focuses on uncertainty.

Various reasons for reluctance of including meta-data about information maturity into engineering design practice were also mentioned in the literature. One is the effort to add this metadata without instant gratification: For example, Culley et al. (2005) state: "Assessing the quality of information is also an arduous process that adds to engineers' workloads". It was also noted that most of the few publications relating states of design information maturity to potential design process improvements, such as strategies for organising tasks, are theoretical in nature, with limited empirical foundation.

\subsection{Insights from a practitioner interview study}

Complementing the literature review, an interview study was undertaken to better appreciate the challenges faced by practitioners in handling immature information in the engineering design process. Case Study Research was carried out following Yin (1994). Insights about practitioners' experience and challenges working with preliminary design information were revealed in semi-structured and linked Interviews with 15 participants at four companies in New Zealand. A total of more than 18 hours of interview data were recorded. Space restrictions prevent more detailed discussion in this publication.

Some important findings from the interviews are summarised here. Firstly, the interviews confirmed the need for practitioners to work with preliminary information and confirmed that challenges can arise from misunderstanding the maturity of that information. Secondly, it was determined that while individual engineers can benefit from an appreciation of information they are working with, many decisions that could be informed by understanding information maturity, such as how to overlap and prioritise work, are owned by project managers. Thirdly, it was found that a confusion about how to describe different aspects of maturity exists. Different interview participants tended to use several terms to describe one facet of maturity. At the same time, one term might be used to describe different issues. Finally, while discussing possible solutions to help with managing and communicating information maturity in design practice, it was found that any support approach would need to be easy to use.

\subsection{Concept for a support approach}

Overall, the interview study and literature review informed the development of a concept for a support approach, which is the main topic of the paper. The approach is depicted in overview in Figure 1 and discussed below.

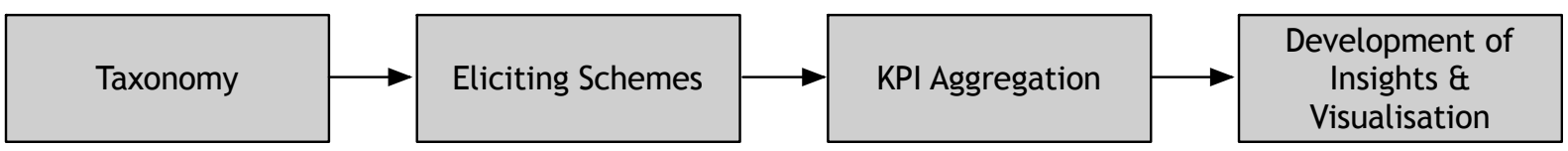

Figure 1. Overview of the approach proposed in this paper

Firstly, the foundation for the approach is a hierarchically structured taxonomy developed to provide internally consistent and sharp definitions of different aspects of information maturity in design. The taxonomy is necessary to clarify the profusion of terms encountered in the literature review and interviews. 
Secondly, practitioners are to be supported in assessing multiple key facets of information maturity, using an eliciting scheme whose structure is equivalent to the taxonomy.

Thirdly, noting that many items of information could be assessed during a project and from different perspectives (e.g. producer and consumer of the information), an aggregation algorithm is used to summarise overall information maturity based on the multiple assessments of different maturity facets. Fourthly, approaches are needed to generate insights from the aggregated assessment results that can help to support the engineering design process. Visualisation approaches are needed to communicate these insights. In this paper, a prototype Augmented Reality (AR) visualisation of insights is presented.

In the following sections, each of these elements of the approach being developed is discussed in turn.

\section{Taxonomy}

A taxonomy was developed based on literature review to define and organise the different facets of preliminary information maturity, in order to lay the foundation for the approach described above.

The literature review revealed that taxonomies of information maturity types and the related field of uncertainty types have been proposed by several authors. Uncertainty plays an important role in decision making and risk management (Thunnissen, 2005). Research by Blanco et al. (2007) and Grebici (2007) concerning maturity in engineering design especially focuses on the social aspects of sharing information, accounting for the differences in the sender and receiver perspectives on maturity. The review revealed that definitions of the same terms offered by different authors are at times contradicting. Different interpretations are driven in part due to the intended application or analysis, see for example the discussions of Ambiguity by Schrader et al. (1993), Stacey and Eckert (2003), and Terwiesch et al. (2002).

To resolve these issues, we developed a hierarchical taxonomy structure integrating key concepts relevant to the intended use case of our approach, namely that the terms in the taxonomy would capture the different ways that design information can be preliminary, from the perspective of the person who creates and communicates that information. The taxonomy is intended to be internally consistent, and to achieve this, some terms had to be used in different ways than some authors use them in the literature.

The developed taxonomy is based on the principle that design information maturity has multiple facets which can be assessed independently. At the top level of the taxonomy, these facets of maturity are organised into three categories relating to the Content, Context and Provenance of the information. The distinction of Content and Context is similar to Eversheim et al. (1997)'s uncertainty classification. Provenance is additionally included to account for the received quality or as Browning et al. (2002) summarise: "no metric is better than the data used to calculate it". Within these three categories, a total of 17 distinct facets of maturity were developed and defined with reference to the literature. A subset of the developed taxonomy is summarised in Figure 2, with focus on the facets of information maturity that are needed to appreciate the rest of this paper. These facets are each discussed in the next subsections.

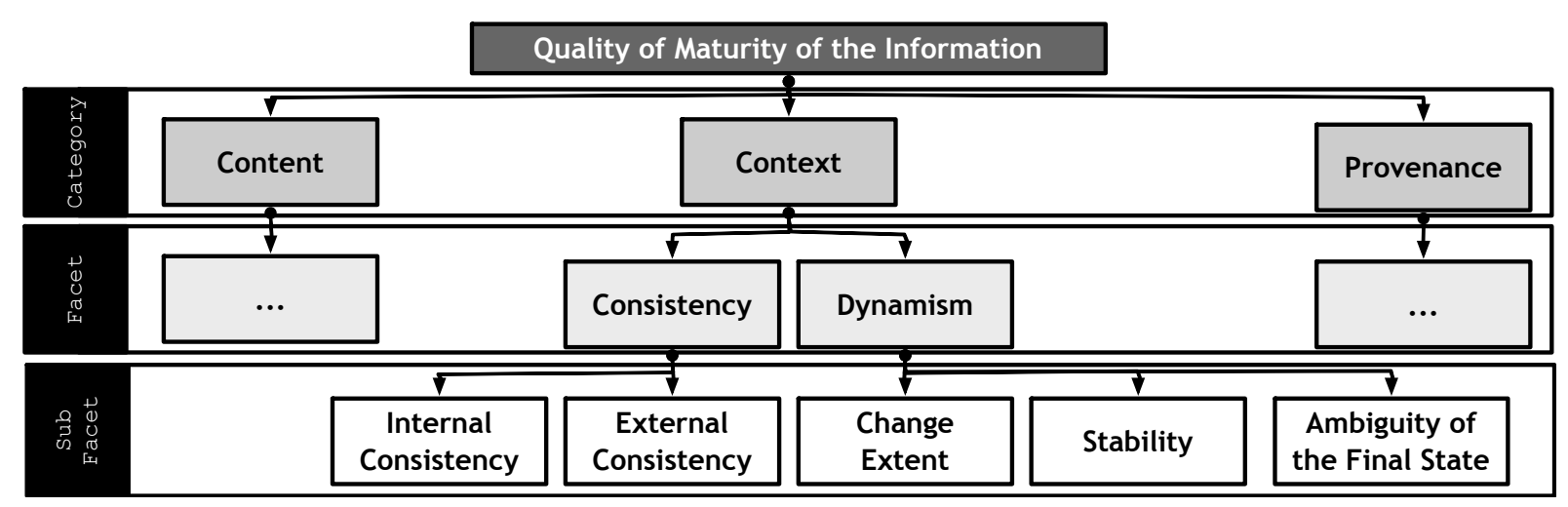

Figure 2. Taxonomy overview emphasising facets of maturity considered in this paper 


\subsection{Consistency}

High consistency of preliminary information assists concurrency in the design process and to avoid unnecessary rework when work is done based on inconsistent information. Maximum consistency is reached if a single source of truth exists. The taxonomy considers that inconsistency can arise within the information or between the information and its surroundings, leading to two distinct facets:

Internal Consistency is defined as how well the content of the information is consistent within itself. Miller (1996) provides several reasons for low consistency (which he terms coherence): Irrelevant Detail, Confusing Measure, and Ambiguous Format. An example of low consistency is the references to section numbers within a document not being correct, or the concepts being discussed in different sections not being in accord. Consistency could be improved, in this case, by proof-reading and editing the document.

External Consistency is defined here as to how well the information meshes with its environment and other related information. Miller (1996) uses the term Compatibility for this and states "No information is an Island [...] Information quality lies not only in the quality of the information itself, but also in how it can be combined with other information [...] this often involves systems working together". An example cause of external inconsistency is design changes made within an FEA not being fed back into the CAD file it was derived from. External consistency might be improved by careful coordination among steps within the project process. Wynn et al. (2011) advise paying close attention to stages prone to cause inconsistencies such as translations, simplification, concurrency, potentially outdated iteration results exist or where a general lack of overview is likely.

\subsection{Dynamism}

Complementing consistency, dynamism relates to the change in the preliminary information over time. In the simplified taxonomy it comprises three facets:

Change Extent represents the anticipated 'amplitude' of future change in the information before it reaches its final state.

Stability is defined by Terwiesch et al. (2002) as the "likelihood of [the preliminary information] no longer changing through the remainder of the process". Stability can also be conceptualised as the frequency or rapidity of expected future change in the information.

Ambiguity of the Final State represents how well-understood the final state of the information is. This is important because maturity is relative to the final state. If the final state is not well understood, any assessment of how close the information is to that state is likely to be inaccurate. To illustrate, one participant in the interview study used the metaphor that encountering problems while project managing a design team is at times similar to cycling in the fog where it is difficult to tell whether an upcoming inclination was approaching a rolling hill or a foothill of the Kilimanjaro. For example, one contributor to ambiguity of the final state is unclear requirements and/or difficulty translating requirements into technical specifications, leading to a design task that might change over time.

\section{Scheme for eliciting the maturity of design information}

The eliciting schemes presented in this section operationalise the theoretical constructs of the taxonomy to help engineering design practitioners assess the maturity of information they create during their day-to-day practice. A maturity grid approach is used. To minimise as far as possible the subjectivity of the assessment, not only a scale is provided but also statements of how to interpret the available levels are presented. The assessor is asked to circle the statement that best describes their assessment of the current situation, which corresponds to a numeric value.

During development of the eliciting scheme, it was determined that different types of information used during design require different interpretations of the maturity facets discussed in the previous section. The maturity grid was therefore customised depending on the type of information that is being assessed. Three grids were developed for three commonly-used types of design information indicated by Dym (1994): a parameter value, a document, a CAD file.

Influenced by the descriptions of Clarkson and Hamilton (2000) regarding design experts' preference of choosing between several values to assess their confidence in design parameters, a discrete interval 
scale was chosen. Originally, it was intended to provide three options by analogy to a traffic light representing a high/medium/low level of maturity against each facet. However, during initial tests where the author attempted to apply the approach to assess the maturity of this research work, it was found that intermediary values should be made available to provide sufficient differentiation during assessments. The scale was accordingly adjusted to $1-5$, with 5 representing the best outcome.

To illustrate the general approach, Figure 3 shows an excerpt from the maturity grid for a document, showing only the facet change extent as defined above. To assess the maturity for a particular item of design information, the person who generates and communicates that information should circle the most appropriate description. Repeating this process for other rows in the grid yields a set of values that together express the maturity of the information.

\begin{tabular}{|l|l|l|l|l|l|l|}
\hline Maturity* & Document & 5 & 4 & 3 & 2 & 1 \\
\hline $\begin{array}{l}\text { DYNAMISM } \\
\text { Maturity relating } \\
\text { to anticipated } \\
\text { future change in } \\
\text { the preliminary } \\
\text { information }\end{array}$ & $\begin{array}{l}\text { Change Extent } \\
\text { Do I expect the } \\
\text { information to } \\
\text { change } \\
\text { substantially or } \\
\text { just marginally? }\end{array}$ & $\begin{array}{c}\text { This document is } \\
\text { almost final. Only } \\
\text { minor changes like } \\
\text { orthographic } \\
\text { corrections etc. are } \\
\text { expected }\end{array}$ & $\begin{array}{c}\text { Until the end of the } \\
\text { project, I expect some } \\
\text { sections to be moved } \\
\text { around and rewritten in } \\
\text { part. }\end{array}$ & $\begin{array}{c}\text { Many sections can only } \\
\text { be added after several } \\
\text { awaited inputs are } \\
\text { received. The current } \\
\text { document is expected } \\
\text { to change } \\
\text { substantially. }\end{array}$ \\
\hline
\end{tabular}

Figure 3. Circling assessment example

\section{Scheme for aggregation of maturity assessments}

Performing the assessments described above leads to a large number of values for each information item assessed. Each information item may have up to 17 numbers (for the full taxonomy) or five items (for the simplified taxonomy presented in this paper). A method is therefore needed to aggregate these assessed values to provide an overall picture of the maturity of an item of information.

Several approaches could be envisaged. To illustrate, Antonsson and Otto (1995) broadly classify compensating and non-compensating aggregations. A weighted sum is a compensating aggregation because a high value on one criterion can compensate for a low value on another. Antonsson and Otto (1995) stress the importance of using non-compensating aggregations for the context of engineering design where safety-relevant features or minimum requirements cannot simply be traded-off as proposed by Utility Theory. Multiplications are a common means of achieving this because a low value or even zero in one category substantially impacts the result. For compensating aggregations Antonsson and Otto (1995) promote the use of fuzzy sets to gain a more realistic impression of customer requirements. For example, full customer satisfaction by a device's minimum range of 250 $\mathrm{km}$ is not rendered zero if the achieved range is only $249 \mathrm{~km}$. They advise for the situation-specific exploration of the true customer wish. The benefits of employing fuzzy algorithms lie in achieving more formal trade-off functions which are more easily expressed in linguistic terms by practitioners.

Another possibility of aggregating KPIs in a meaningful way for the specific context of this paper is found in the work of Ballou and Pazer (2003). They condense the aspects of completeness and consistency of information into the utility of that information for a decision-maker (U) by including their preference for completeness over consistency (p), as shown in Equation (1):

$$
\mathrm{U}=\mathrm{p} * \text { Completeness }+(1-\mathrm{p}) * \text { Consistency }
$$

The aggregation approach adopted for this paper incorporates several of the aforementioned possibilities into a newly developed aggregation scheme shown in Figure 4. For an item of information being assessed, the outcome of the maturity assessment is five dimensions, each specified on a scale of 1-5. The approach of combining values in a meaningful way to one aggregated maturity value is shown in Figure 4 and based on the following logic:

- Considering the aggregation of Internal Consistency (F1A) and External Consistency (F1B), these are interpreted as complementary because a document meshing perfectly with itself but not integrated into its context does not imply a summarised good level of Consistency. A noncompensating aggregation function - implemented with the multiplication after normalisation to an $[0,1]$ interval - is therefore used in accordance with Ballou and Pazer (2003). 
- Considering the aggregation of Change Extent (F2A) and Stability (F2B), the relative importance of these facets is heavily influenced by the downstream tasks ability to incorporate changes caused by these sub-facets without causing significant rework. Krishnan et al. (1997) give an example in which larger changes in upstream information are likely to induce more significant iteration in downstream tasks, while frequent changes have also been said to potentially increase the amount of rework (Wynn et al., 2011). For this paper Change Extent (F2A) and Stability (F2B) are therefore deemed equally important to information maturity, with a compensating relationship and therefore are averaged.

- Considering the aggregation of Ambiguity of the Final State (F2C) with the result of the previous bullet point, it was noted earlier that if the final state is poorly understood, it is likely that many of the other assessments will be inaccurate and hence, overall maturity should be low. Therefore, for this example, three times higher importance is assigned to $\mathrm{F} 2 \mathrm{C}$ than to the average of F2A and F2B, using the weight of $3 / 4$.

- Finally, we allocate the same importance to consistency and dynamism, so these aggregated values are averaged to yield an overall maturity assessment.

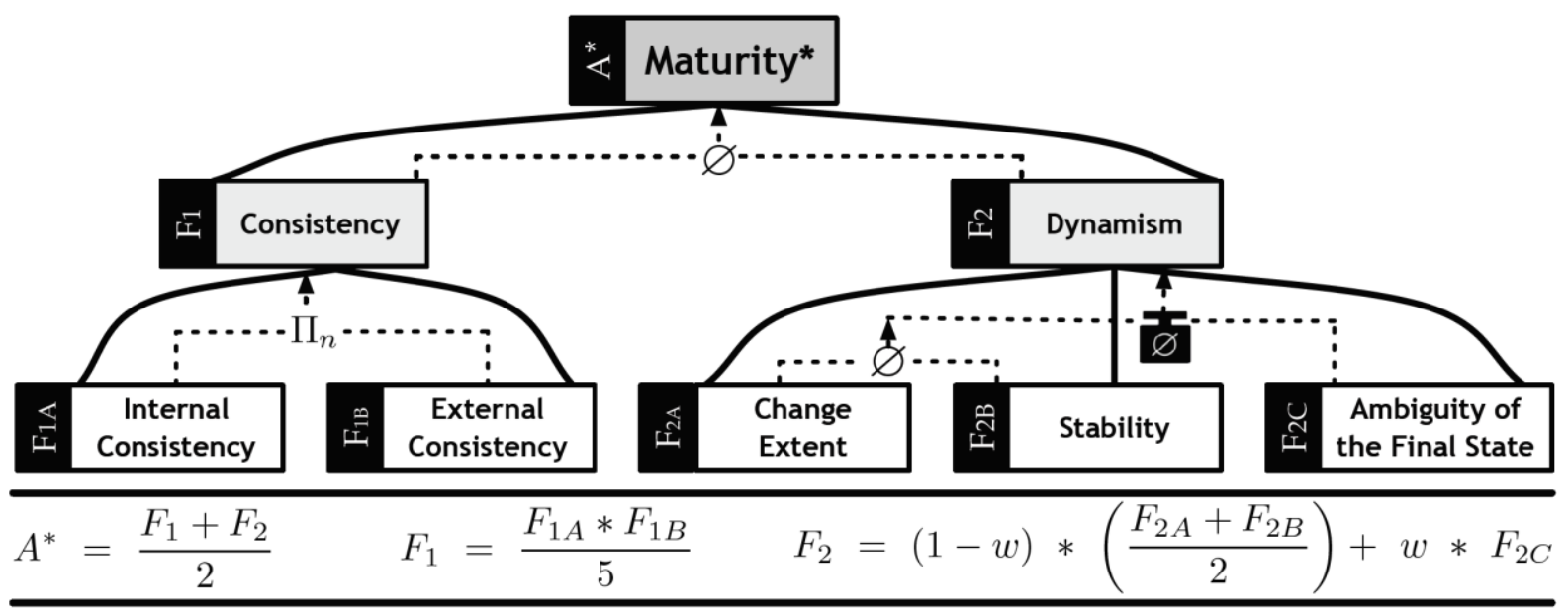

$$
\begin{array}{rlrlll|lr}
\text { Example: } & F_{1 A} & F_{1 B} & F_{2 A} & F_{2 B} & F_{2 C} & w & \text { simple average }=60 \% \\
3 & 4 & 1 & 5 & 2 & 3 / 4 & A^{*}=46.5 \% \\
& A^{*}=\frac{\left[\frac{3 * 4}{5}\right]+\left[\frac{1}{4}\left(\frac{1+5}{2}\right)+\frac{3}{4}(2)\right]}{2}=2.325 &
\end{array}
$$

\section{Figure 4. Aggregation scheme for maturity}

The example with some illustrative numbers, as shown in Figure 4, shows that uniformly averaging these five maturity facets results in a $60 \%$ aggregated Maturity value. The adjusted A* aggregation puts stronger emphasis on the relatively low values for F2A, and F2C resulting in 46.5\% aggregated maturity level. Overall, the advantage of this approach is to reduce the occluding effect of aggregation. The above explanation concerns how the different elements that are assessed for a single item of information can be aggregated into a single maturity indicator. Additionally, multiple information items may be associated with a single component or subsystem at any time during design, and the same information might conceivably be assessed by several individuals who participate in generating it. It is proposed that in such cases, the $\mathrm{A}^{*}$ values for different assessments relating to a particular component or subsystem of the design be averaged to yield an overall assessment of maturity.

\section{Development of insights and visualisation}

The final step in the approach concept is to use the aggregated maturity assessments to develop insights to support the engineering design process. The insights also need to be visualised and communicated. 


\subsection{Development of insights}

The maturity information could potentially provide insights for practice in various ways, ranging from tracking the status of a project, to identifying parts undergoing iteration, to simply making engineers aware of the maturity status of information they are using as input for their tasks. This section focuses on a specific use case: providing dynamic, context-aware guidance regarding which components or subsystems in a project might need special attention from management at any given time. This is achieved by combining the theoretical insights of Maier et al. (2014) with the actual information maturity as might be assessed in a real project, and a design structure matrix (DSM) that captures connectivity between subsystems in the emerging design.

As background, Maier et al. (2014) use simulation of several cases and scenarios to show that several generic work priority rules can be used to improve performance of an engineering design process, considering how different subsystems evolve over time during the design process. They recommend various policies for prioritising work to avoid unnecessary design iterations. Here we focus on three of these policies, whose implementation in practice would require knowledge about the architecture of the design and the way that maturity of each subsystem is evolving during the project:

1. Their first policy "Lowest passive sum first" (M1) is based on a DSM capturing the connections between project subsystems. In essence, they recommend that subsystems which are least dependent on other tasks' feedback be prioritised for completion, because they are least likely to be invalidated by such feedback.

2. Their second policy "Highest maturity level first" (M2) states that subsystems that currently possess a low maturity level in relation to others should not be a particular focus of attention. This is because when one subsystem has relatively low maturity, it is usually because it has suffered from rework imposed on it by other subsystems. Hence, concentrating effort on increasing maturity in such a subsystem is likely to result in that effort being later invalidated.

3. Their third policy "least reworked first" (M3) similarly states that if a system has received much rework recently, effort would be better spent on resolving the component driving the rework.

The concept presented in this section is to use these related insights to inform management of which subsystems in a project may need to be prioritised for special attention, considering the evolving maturity status of all the subsystems as elicited using the scheme of Section 4 and aggregated as per Section 5. In other words, the guidance is dynamic and responsive to practitioners' assessments of the current project status.

The approach taken is to compute a priority score for each component in an emerging design using the scheme presented in Figure 5. In overview, the KPIs defined in the figure, referred to as K1-K5, are all calculated for each component using the formulae shown in the column 'KPI \& formula'. For each KPI, the depicted rules are then used to convert the KPI value into a penalty factor, as explained in detail below. The penalties associated with all five KPIs are finally summed up to generate a priority score of the component being assessed. The KPIs are discussed in turn below.

- K1. This KPI accounts for policy M1 described above. The number of inputs to each component is calculated from the DSM. These values are then classified into three categories (low/medium/high) leading to a penalty of 0,6 or 9 respectively. As we assign a high priority to M1, the aforementioned classification implies a three times higher penalisation (i.e. up to a penalty of nine for the worst outcome) than some of the others.

- K2. This KPI accounts for policy M2 by considering the aggregated maturity associated with each component (whose computation was presented in Section 5) as assessed on the ordinal 1-5 scale introduced in Section 4. A result significantly lower than the central value of 3 (i.e. <2) leads to a high penalty. In this example, a value equal or higher than 4.8 leads to a penalty of 0 .

Finally, M3 is incorporated by including the dynamism of the information in the priority recommendations. This is achieved through three further key performance indicators (KPI), whose lower hierarchical value is accounted for by assigning a lower maximum penalty: 
- K3. This captures the recent history as the difference between the previous and current value for stability. A deterioration in maturity- given by a difference of less than zero - shall entail a penalty of 3 .

- K4. Change extent and stability shall be averaged for the same reasons stated around Figure 4.

- K5. Ambiguity of the final state is also included---the lower this aspect of information maturity, the higher the corresponding penalty.

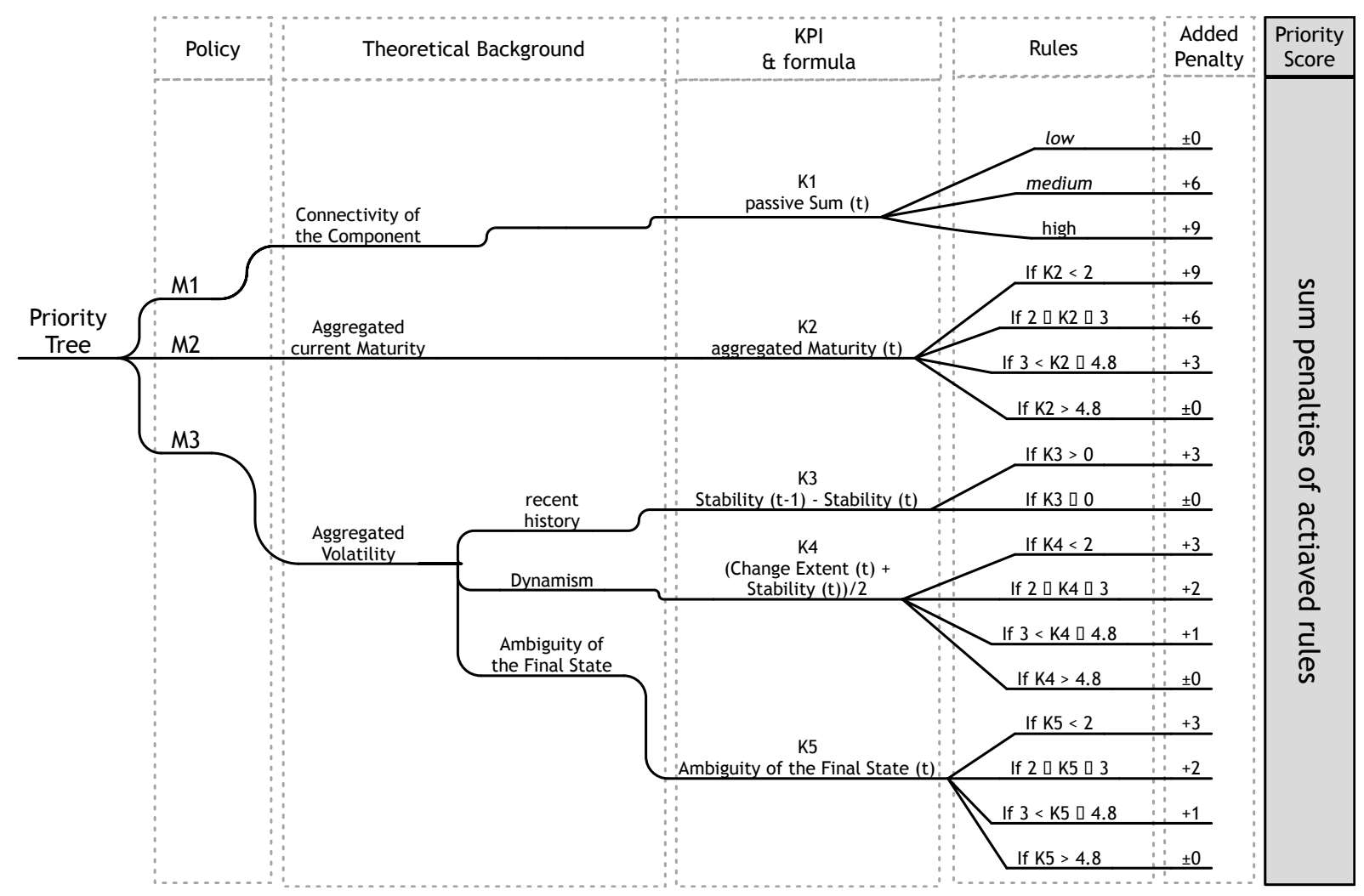

Figure 5. Logic Tree of the Priority Score

Summing the penalties generated from K1-K5 generates the overall priority score for each component. The lower the score, the more the component can be recommended for attention according to the conclusions of Maier et al. (2014).

To summarise, the computations described above generate high-level recommendations on which parts of a project may deserve prioritisation from the information maturity assessments that could, in principle be completed by individuals working on the project. The required inputs are a DSM showing connections between subsystems, the maturity assessment results relating to each subsystem. In principle, these recommendations might be regularly updated to provide up-to-date advice that responds to the state of the project.

\subsection{Visualisation}

The final step in the proposed approach concept is to visualise the insights for presentation to the project team. Such a presentation could be achieved in various ways. Though closely-involved design experts are likely to be working on CAD systems which could arguably be enhanced by the integration of maturity status, an Augmented Reality (AR) visualisation was chosen. This requires a physical prototype of a product being available that can be enhanced by overlaying virtual content. Apart from the trend of digital twinning, increased prototyping is reported, for example, Tahera et al. (2017) show a case where an outdated version of the product forms the foundation for the development of the next generation of product, termed the "Mule". Iteratively old parts are exchanged for new parts. In conjunction with a remark of an interviewee, where placeholders of the outdated version were highlighted in bright pink within the CAD assembly, we develop our AR experience. 
In Figure 6, two use-cases are presented based on some hypothesised data for illustration purposes. On the left, an intuitive virtual false-colour overlay based on traffic-lights is used to indicate aggregated Maturity levels which are derived following the computation shown in Figure 4. One expected group of beneficiaries are project participants who might work on their individual parts and are empowered with a visualisation of the maturity status of their as well as surrounding parts.

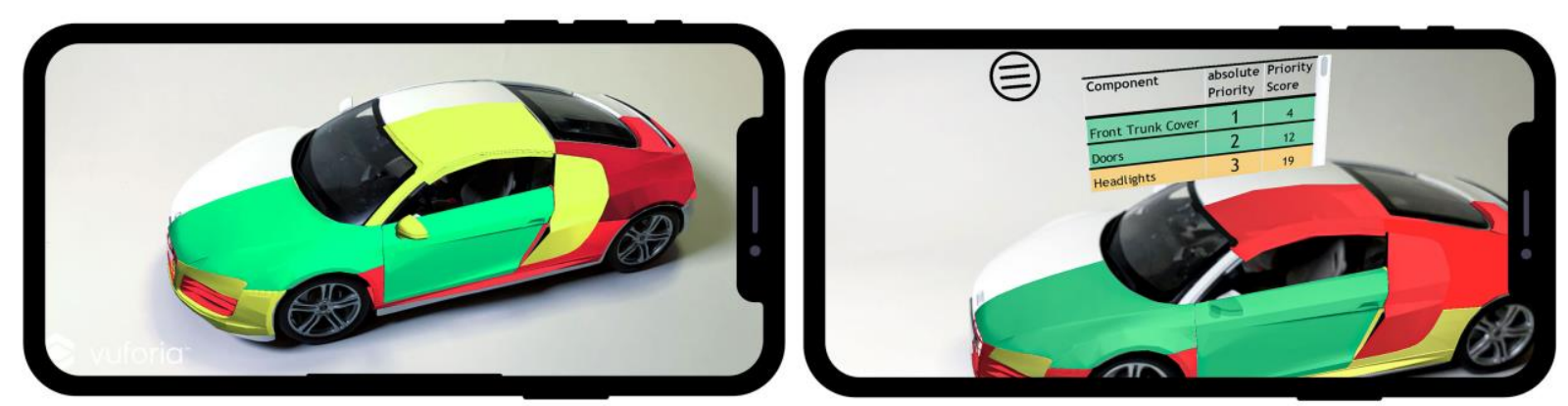

Figure 6. Visualisation of the aggregated maturity levels (left) and subsystem priorities (right)

The right side of Figure 6 shows a similar visualisation, but now priorities for each subsystem as computed in Section 6.1 are shown. As well as the colour-coding, advice about prioritising the subsystems is given in form of a sorted pop-up table.

As this concept-demonstrator only requires users with a smartphone, it has potential to support design and management reviews resolving some of the challenges mentioned in the introduction.

This decision of using AR is supported by the authors experience of hosting non-co-located and interdisciplinary design meetings where visualisations outside of the usual CAD environment tend to increase participation especially of non-experts. Including their remarks has potential for a large added benefit.

\section{Outlook and conclusion}

Engineering design and its management could be supported by assessing the maturity of preliminary design information at different points during a project. Discussed in this paper, a literature review and interview study has indicated the importance of this topic and pinpointed a research gap. A concept for an approach to address this gap has been presented. The ideas presented here represent progress partway through a $\mathrm{PhD}$ project, and much remains to be done to fully concretise and validate the approach.

In future refinements, we intend to further detail the taxonomy, explore and improve the aggregation approaches, and enhance the IT support so that the approach can be tested in an industrial context.

The intention is to provide a concept that in principle, would be usable for practitioners during their work. The combination with a PLM system might provide the ability to automatically trigger situation-specific warnings or workflows. The availability of detailed maturity data resulting from these assessments could also provide a data set from which insights about the relation between information maturity and design progress could be extracted.

\section{References}

Antonsson, E.K. and Otto, K.N. (1995), "Imprecision in Engineering Design", Journal of Mechanical Design, Vol. 117 No. B, pp. 25-32. https://doi.org/10.1115/1.2836465

Ballou, D.P. and Pazer, H.L. (2003), "Modeling completeness versus consistency tradeoffs in information decision contexts", IEEE Transactions on Knowledge and Data Engineering, Vol. 15 No. 1, pp. 240-243. https://doi.org/10.1109/TKDE.2003.1161595

Blanco, E., Grebici, K. and Rieu, D. (2007), "A unified framework to manage information maturity in design process", International Journal of Product Development, Vol. 4 No. 3/4, pp. 255-279. https://doi.org/ 10.1504/ijpd.2007.012495

Browning, T.R. et al. (2002), "Adding value in product development by creating information and reducing risk", IEEE Transactions on Engineering Management, Vol. 49 No. 4, pp. 443-458. https://doi.org/10.1109/ tem.2002.806710 
Clarkson, P.J. and Hamilton, J.R. (2000), "Signposting, A Parameter-driven Task-based Model of the Design Process", Research in Engineering Design, Vol. 12 No. 1, pp. 18-38. https://doi.org/10.1007/s001630050021

Culley, S.J. et al. (2005), "An assessment of quality measures for engineering information sources", ICED 05: 15th International Conference on Engineering Design. Barton, Australia, 2005, Engineers Australia, pp. 443-456.

Dym, C.L. (1994), "Representing designed artifacts: The languages of engineering design”, ARCO 1994, Vol. 1, pp. 75-108. https://doi.org/10.1007/bf02736181

Eversheim, W. et al. (1997), "Information management for concurrent engineering", European Journal of Operational Research, North-Holland, Vol. 100 No. 2, pp. 253-265. https://doi.org/10.1016/s03772217(96)00288-3

Grebici, K. (2007), La maturité de l'information et le processus de conception collaborative, [PhD Thesis], Institut National Polytechnique de Grenoble.

Krishnan, V., Eppinger, S.D. and Whitney, D.E. (1997), “A Model-Based Framework to Overlap Product Development Activities", Management Science, Vol. 43 No. 4, pp. 437-451. https://doi.org/10.1287/ mnsc.43.4.437

Maier, J.F. et al. (2014), "Simulating progressive iteration, rework and change propagation to prioritise design tasks", Research in Engineering Design, Vol. 25 No. 4, pp. 283-307. https://doi.org/10.1007/s00163-0140174-8

Miller, H. (1996), "The multiple dimensions of information quality", Information Systems Management, Vol. 13 No. 2, pp. 79-82. https://doi.org/10.1080/10580539608906992

Schrader, S., Riggs, W.M. and Smith, R.P. (1993), "Choice over uncertainty and ambiguity in technical problem solving”, Journal of Engineering and Technology Management, Vol. 10 No. 1-2, pp. 73-99. https://doi.org/ 10.1016/0923-4748(93)90059-r

Stacey, M. and Eckert, C.M. (2003), “Against Ambiguity”, Computer Supported Cooperative Work (CSCW), Vol. 12 No. 2, pp. 153-183. https://doi.org/10.1023/a:1023924110279

Tahera, K., Earl, C. and Eckert, C.M. (2017), “A Method for Improving Overlapping of Testing and Design”, IEEE Transactions on Engineering Management, Vol. 64 No. 2, pp. 179-192. https://doi.org/10.1109/ tem.2017.2654223

Terwiesch, C., Loch, C.H. and De Meyer, A. (2002), "Exchanging Preliminary Information in Concurrent Engineering: Alternative Coordination Strategies", Organization Science, Vol. 13 No. 4, pp. 402-419. https://doi.org/10.1287/orsc.13.4.402.2948

Thunnissen, D.P. (2005), Propagating and mitigating uncertainty in the design of complex multidisciplinary systems, [PhD Thesis], California Institute of Technology.

Wynn, D.C., Grebici, K. and Clarkson, P.J. (2011), "Modelling the evolution of uncertainty levels during design", International Journal on Interactive Design and Manufacturing (IJIDeM), Vol. 5 No. 3, pp. 187202. https://doi.org/10.1007/s12008-011-0131-y

Yin, R.K. (1994), Case Study Research: Design and Methods, Sage Publications, Newbury Park, California, USA. 